\title{
Voltage Graphs, Group Presentations and Cages
}

\author{
Geoffrey Exoo \\ Department of Mathematics and Computer Science \\ Indiana State University \\ Terre Haute, IN 47809 \\ g-exoo@indstate.edu
}

Submitted: Dec 2, 2003; Accepted: Jan 29, 2004; Published: Feb 14, 2004

\begin{abstract}
We construct smallest known trivalent graphs for girths 16 and 18. One construction uses voltage graphs, and the other coset enumeration techniques for group presentations.
\end{abstract}

AMS Subject Classifications: 05C25, 05C35

\section{Introduction}

The cage problem asks for the construction of regular graphs with specified degree and girth. Reviewing terminology, we recall that the girth of a graph is the length of a shortest cycle, that a $(k, g)$-graph is regular graph of degree $k$ and girth $g$, and that a $(k, g)$-cage is a $(k, g)$-graph of minimum possible order. Define $f(k, g)$ to be this minimum. We focus on trivalent (or cubic) cages. It is well known that

$$
f(3, g) \geq \begin{cases}2^{g / 2+1} & \text { if } g \text { is even } \\ 3 \times 2^{(g-1) / 2}-2 & \text { if } \mathrm{g} \text { is odd }\end{cases}
$$

This bound, the Moore bound, is achieved only for girths 5, 6, 8, and $12[1,4]$. The problem of finding cages has been chronicled by Biggs [3] and others. In this note we give two new constructions of cage candidates using different methods. 


\section{A Girth 16 Lift of the Petersen Graph}

The first, and simplest, of the constructions begins with the Petersen graph (denoted $P$ ), the smallest 3-regular graph of girth 5 . We investigate graphs that can be constructed as lifts of the $P$, and discover a new graph, the smallest known trivalent graph of girth 16. Note that lift have been previously used with reference to the closely related degreediameter problem [9]. The graph has 960 vertices, improving the old bound of 992 given in [2]. Our method is best described using voltage graph terminology, first introduced by Gross [7], which we now review.

If $G$ is a finite graph, denote its vertex and edge sets by $V(G)$ and $E(G)$. Also let $D(G)$ denote the set of arcs on $G$ : each edge $e \in E(G)$ is represented exactly twice in $D(G)$, i.e., with each of the two possible orientations. If $e \in D(G)$, we denote the reverse arc by $e^{-1}$. If $\Gamma$ is a finite group, a voltage assignment is a function $\phi: D(G) \rightarrow \Gamma$, such that if $\phi(e)=g$ then $\phi\left(e^{-1}\right)=g^{-1}$. Given a voltage assignment, the lift of $\mathrm{G}$ in $\Gamma$ via $\phi$, denoted $G_{\phi}$, is the graph whose vertex set is $V(G) \times \Gamma$ in which $(u, g)$ and $(v, h)$ are adjacent if $u v \in E(G)$ and $g \cdot \phi(u v)=h$. The projection map $\pi_{\phi}: G_{\phi} \rightarrow G$ is defined by $\pi_{\phi}(v, g)=v$.

Since we are dealing with questions of girth, we are interested in identifying cycles that occur in lifts. In turns out that cycles in a lift correspond to a certain type of walk in the base graph. Recall that a walk in a graph is an alternating sequence of vertices and edges, beginning and ending with a vertex, in which each element in the sequence is incident with the elements before and after it. A closed walk is a walk that begins and ends with the same vertex. A non-reversing walk is a walk in which no vertex is preceded and followed by the same edge.

Observation 1. Let $G_{\phi}$ be a lift of a graph $G$ with voltage assignments in the group $\Gamma$. If $v_{1}, \ldots, v_{k}$ are the vertices of a $k$-cycle in $G_{\phi}$, then $\pi_{\phi}\left(v_{1}\right), \ldots, \pi_{\phi}\left(v_{k}\right)$ are the vertices of a closed non-reversing walk in $G$ such that the product of the voltage assignments along the walk is the group identity.

In the case where the lifting group is abelian, the girth of the lift is limited. Recall that a theta graph is a graph consisting of two vertices of degree three joined by three independent paths.

Given a theta graph, it is easy to find a closed non-reversing walk in which each edge is traversed twice, once in each direction. Hence for any voltage assignment from an abelian group, the product along the walk is the identity. So we have the following.

Observation 2. Let $G$ be a graph which contains a subgraph isomorphic to a theta graph. If the theta-subgraph contains $t$ edges, then the girth of a lift of $G$ via an abelian group is at most $2 t$.

Since the Petersen graph contains theta graphs with eight edges, any lift via an abelian group has girth at most 16. It may be somewhat surprising that girth 16 can be achieved.

The following table gives the numbers of closed non-reversing walks in the Petersen graph for each of the lengths relevant to our construction. 


\begin{tabular}{|r|r|}
\hline Length & Number \\
\hline \hline 3 & 0 \\
4 & 0 \\
5 & 12 \\
6 & 10 \\
7 & 0 \\
8 & 15 \\
9 & 20 \\
10 & 66 \\
11 & 120 \\
12 & 140 \\
13 & 300 \\
14 & 570 \\
15 & 1104 \\
\hline Total & 2357 \\
\hline
\end{tabular}

Table. Closed non-reversing walks in the Petersen graph.

So if we are trying to construct a lift of girth 16 using a nonabelian group, our voltage assignment must satisfy a total of 2357 inequalities. For abelian groups this number can be reduced by about $2 / 3$.

Our construction employs the group $Z_{48} \times Z_{2}$, and proceeds as follows. First select a vertex $v_{0} \in V(P)$ and assign the identity to all edges on the tree of radius two centered at $v_{0}$. It remains to label the edges of a hexagon (see the Figure). While it may be possible to find a solution by hand, it is a simple matter to find one via computer search. In fact, one can find all solutions in about a minute on a fast PC. One such solution is depicted in the Figure. We found that all solutions produce the same graph.

The reader may be curious about the choices made for the base graph and the lifting group. The choice of the Petersen graph is a fairly obvious one. In fact, we systematically looked for lifts of the Petersen graph of various girths. In the process, we learned a little about which groups work well. A small example may illustrate the point.

Suppose we wish to construct a girth 8 lift of the Petersen graph. Since the 8-cage has 30 vertices, is the unique trivalent graph of girth 8 , and is not a lift of $P$, the smallest candidate will have 40 vertices. In this case there are two choices for the lifting group, $Z_{4}$ and $Z_{2} \times Z_{2}$. It turns out that $Z_{4}$ does not work, and it is instructive to understand why $Z_{2} \times Z_{2}$ does work.

Once again we consider a distinguished vertex $v_{0}$ of $P$, and label all edges in the binary tree of radius two centered at $v_{0}$ with the identity. As above, it remains to assign group elements to the six edges of the outer 6-cycle. Denote the elements so assigned by $g_{i}$, $0 \leq i \leq 5$, in cyclic order.

Since the Petersen graph contains no 7-cycles, we only need to insure that the lift contains no 5-cycles and no 6-cycles. Now $P$ contains twelve 5-cycles, six of these consist of four tree edges and one outer edge, and six consist of two tree edges and three consecutive outer edges. So the group assignments for a lift with girth 8 must satisfy $g_{i} \neq 0$ and 


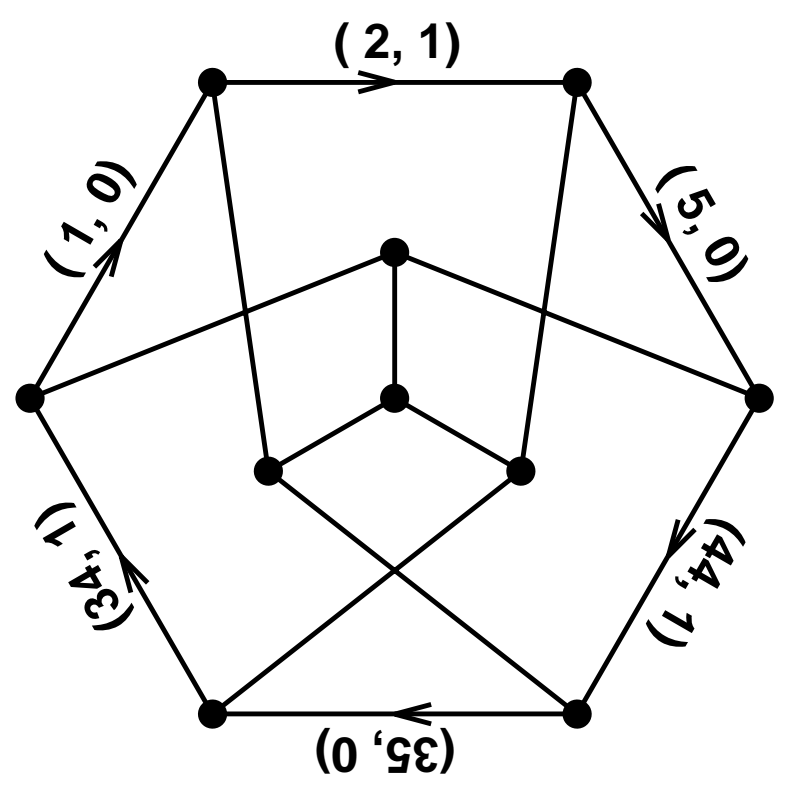

Figure 1: A trivalent graph of girth 16. 
$g_{i}+g_{i+1}+g_{i+2} \neq 0$. Similarly, $P$ contains ten 6 -cycles. Six of these contain two consecutive edges from the outer cycle, so we require that $g_{i}+g_{i+1} \neq 0$. There are also three 6 -cycles that contain opposite pairs of edges from the outer cycle. These edges are traversed in opposite orientations, so we require that $g_{i}-g_{i+3} \neq 0$ (of course in the particular case of $Z_{2} \times Z_{2}$ orientation does not matter). A final 6-cycle is the outer cycle, so we have $\sum_{i=0}^{5} g_{i} \neq 0$. Summarizing, we have

$$
\begin{aligned}
g_{i} & \neq 0 \\
g_{i}+g_{i+1}+g_{i+2} & \neq 0 \\
g_{i}+g_{i+1} & \neq 0 \\
g_{i}-g_{i+3} & \neq 0 \\
\sum_{i=0}^{5} g_{i} & \neq 0
\end{aligned}
$$

Inequalities (1) and (2) insure that there are no 5-cycles, inequalities (3), (4) and (5) eliminate 6 -cycles. We construct a girth 8 lift using voltage assignments from $Z_{2} \times Z_{2}$ as follows. Assign voltages with a first coordinate of 1 to all six outer edges, thereby eliminating 5-cycles. In the second coordinate, we assign alternating values of 0 and 1 , eliminating 6-cycles.

So in this small example we chose a group that was a direct product and used the different factors to eliminate cycles of different lengths. We found similar examples for lifts of girths 9 through 12, and attempted to do a similar thing for girth 16 . We learned that for larger girths the examples do not work out quite so simply. In the end, we looked at several groups (with more direct factors) before finding the group that worked.

\section{A Small Graphical Regular Representation of Girth 18}

When making our second construction we work directly with the generators and relators for certain groups, and look at the Cayley graphs determined by these groups. Our groups are all generated by three involutions. (Biggs [2] calls the Cayley graphs for such groups Type II Cayley graphs). So we look for presentations with three generators, $a, b$, and $c$, the three relators of the $a^{2}=b^{2}=c^{2}=1$, and certain other relators. The other relators who chose are words of length $g$, the girth of the graph we are trying to construct. Of course there is no guarantee that the Cayley graph for such a group presentation, even if finite, will have girth $g$. We are particularly interested in the case $g=18$.

To (attempt to) determine the group given by such a presentation, we use the ToddCoxeter coset enumeration procedure, which is outlined in [10], and in greater detail in [12] where it called the Scheier-Todd-Coxeter-Sims procedure.

The procedure attempts to find all cosets of a specified subgroup $H$ of a group $G, G$ is given by generators and relators and $H$ is given as a subgroup generated by a list of 
words. If $H$ is the identity subgroup, as it is in our application, then the cosets can be identified with the elements of $G$.

In the course of the procedure a coset table is constructed. The length of the table grows, and sometimes shrinks, as the procedure proceeds. Since, by the result of Novikov and Boone, the word problem is undecidable [10], there is no guarantee that Todd-Coxeter terminates. So the coset table may grow without bound. Hence when attempting to construct finite Cayley graphs using this procedure, we may need to give up on a particular group presentation without knowing whether or not it determines a finite group. In designing a search algorithm, it is not immediately clear when to give up, since the coset table may grow to a size greater than the size of the group, but later shrink. So we set an upper bound on the the number of group elements created in the course of the prcedure, if this bound is reached we discard the presentation. For the particular case of $g=18$ we use an upper limit of roughly twice the size of the graph we are trying to construct. The search algorithm that we used was a simple variation of the one described in [5] wherein the objective function was the size of the group (or the upper bound).

We remark that four relators are insufficient to determine a finite Cayley graph of girth 18. If we consider two words as equivalent if one can be obtained from the other by reversal, cyclic permutation, or a permutation of symbols, then there are only 1354 essentially different words of length 18. It is a simple matter, thanks to GAP [6], to check that none of these produce a Cayley graph of girth 18. Perhaps none even determine a finite group?

So we use a presentation with five relators, three specifying that the generators are involutions, and two of length 18. In this case, we one can quickly find Cayley graphs with the desired girth. Our best solution yields a group of order 2640. In addition to $a^{2}=b^{2}=c^{2}=1$, the relators for this group are as follows.

$$
\begin{aligned}
& \text { bcababababcbcbcabc }=1 \\
& \text { cacbcababcbcbabacb }=1
\end{aligned}
$$

For those with access to GAP, the following sequence of commands will generate the group.

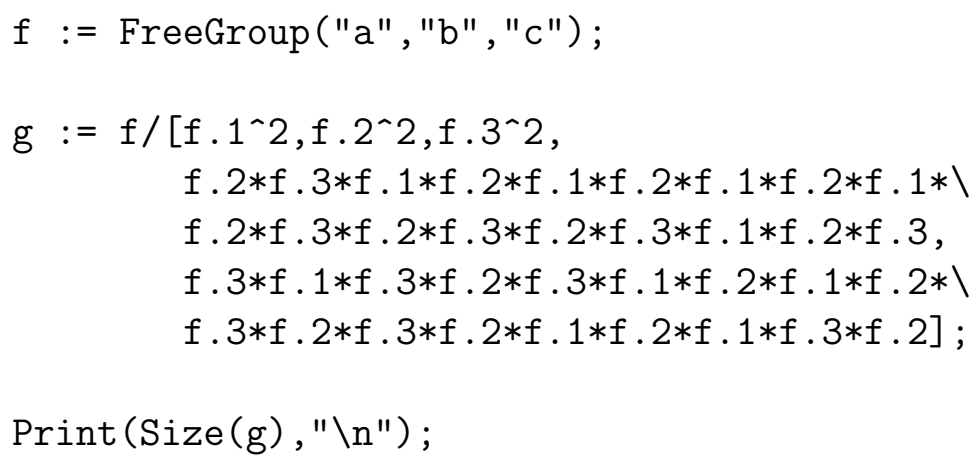

The associated Cayley graph is the smallest known trivalent graph of girth 18 [11]. 
Given the presentation, and a working copy of GAP, it easy to determine other properties. For example, the group element acacbabacbabacacbabacbcb (of length 24) has order 110, and determines a Hamiltonian cycle in the Cayley graph. The element $a b$ has order 110, and the subgroup generated by $a$ and $b$ is the dihedral group of order 220 . If we consider just the $a$-edges and $b$-edges of the graph, we have 12 disjoint cycles of length 220. The $c$-edges connect each of these cycles to exactly five other cycles. From each cycle there are 44 edges to each of five other cycles. If we identify the 220-cycles to vertices, and join identified vertices when the associated cycles are joined by edges, we get an icosahedron. It can be verified, using nauty [8], that the automorphism group of this graph is exactly 2640. Hence the graph is a regular representation.

\section{References}

[1] E. Bannai and T. Ito, On Finite Moore Graphs, J. Fac. Sci. Tokyo, Sect. 1A 20 (1973) 191-208.

[2] N.L. Biggs, Cubic Graphs with Large Girth, Combinatorial Mathematics: Proceedings of the Third International Conference, Annals of the New York Academy of Sciences 555, 1989, 56-62.

[3] N. Biggs, Constructions for cubic graphs of large girth. Electron. J. Combin., 5 (1998) A1.

[4] R. M. Damerell, On Moore Graphs, Proc. Cambridge Phil. Soc., 74 (1973) 227-236.

[5] G. Exoo, Some New Ramsey Colorings. Electronic J. Combinatorics. 5 (1998) \#R29.

[6] The GAP Group, GAP - Groups, Algorithms, and Programming, Version 4.3; 2002, (http://www.gap-system.org).

[7] J. L. Gross, Voltage Graphs. Discrete Math, 9(1974) 239-246.

[8] B. D. McKay, nauty User's Guide, preprint, 1992.

[9] B.D. McKay, M. Miller, and J. Siran, A note on large graphs of diameter two and given maximum degree. J. Combin. Theory Ser. B, 74(1998) 110-118.

[10] J. J. Rotman, The Theory of Groups: An introduction, 3rd. ed., Allyn and Bacon, Inc., Boston, 1965.

[11] G. Royle. (http://www.cs.uwa.edu.au).

[12] A. Seress Permutation Group Algorithms, Cambridge University Press, Cambridge, 2003. 